\title{
Bipolare Störungen Verträgliche Therapieoption bei manischen Episoden verfügbar
}

- Das seit gut einem Jahr zugelassene atypische Antipsychotikum der zweiten Generation Asenapin (Sycrest $^{\circledast}$ ) ist eine Option manischer Episoden in der Monound Kombinationstherapie der bipolaren Störung. Es weist ein vergleichsweise geringes Nebenwirkungspotenzial auf und ist insbesondere zu erwägen bei Patienten, die unter anderen Therapieoptionen mangelnde Wirksamkeit oder schlechte Verträglichkeit zeigen.

Professor Dieter Naber, Hamburg, betonte, dass in der Pharmakotherapie von Patienten mit bipolarer Störung zwischen der Akut- und der Langzeitbehandlung unterschieden werden müsse. Während in der Akutbehandlung die Reduktion von Selbst- und Fremdgefährdung, Erregung, Unruhe und anderer Symptome im Vordergrund stehe, habe die Langzeitbehandlung die Rezidivprophylaxe als Ziel. Lithium und Antikonvulsiva, aber auch atypische Antipsychotika seien sowohl in der Akut- als auch in der Langzeitbehandlung wirksam, allerdings oft mit Verträglichkeitsproblemen verbunden. Die hohe Non-Compliance bipolarer Patienten sei im Wesentlichen bedingt durch mangelnde Krankheitseinsicht und reduzierte Verträglichkeit, so Naber.

Asenapin habe sich in mehreren Kurz- und Langzeitstudien als eine rasch wirksame und gut verträgliche Alternative bei Patienten mit bipolaren Störungen dargestellt. Insbesondere die geringe Inzidenz von rele- vanten Nebenwirkungen wie persistierende Sedierung, Somnolenz, Gewichtszunahme, motorischen Nebenwirkungen und sexuellen Funktionsstörungen sei für die Langzeitbehandlung von wesentlicher Bedeutung.

Naber berichtete von ersten eigenen Therapieerfahrungen mit dem neuen Antipsychotikum. In der Akuttherapie habe er fünf Patienten erfolgreich mit Asenapin (15-20 mg/d) behandelt, alle in Kombination mit Valproat (1.000-2.500 mg/d). Die Wirksamkeit sei ähnlich gut gewesen wie bei anderen Atypika bei den stationären Aufenthalten zuvor. Sowohl objektiv als auch subjektiv seien keine relevanten Nebenwirkungen aufgetreten. In Langzeit behandle er einen Patienten über vier Monate in Monotherapie und einen Patienten über sechs Monate in Kombination mit Valproat. Beide Patienten hätten über eine deutlich bessere subjektive Befindlichkeit berichtet. Sie fühlten sich „normaler“ und verzeichneten weniger Gewichtszunahme.

Asenapin sei insbesondere zu erwägen bei Patienten, die unter Lithium, Antikonvulsiva oder anderen Atypika unzureichende Wirksamkeit und/oder insbesondere mangelnde Verträglichkeit zeigen, resümierte Naber.

Dr. Gunter Freese

Satellitensymposium "Neu bei Manie: Sycrest ${ }^{\circledR}$ - das untypische Antipsychotikum". DGPPN-Kongress, Berlin, 25.11.2011; Veranstalter: Lundbeck GmbH

\section{Therapie der Depression}

\section{Remission ist und bleilbt das Behandllungsziel}

- Mit einer Lebenszeitprävalenz von bis zu 15\% gehört die Depression zu den wichtigsten Volkskrankheiten. Worauf es in der Behandlung affektiver Störungen ankommt, erläuterte Professor Siegfried Kasper, Leiter der Universitätsklinik für Psychiatrie und Psychotherapie in Wien.

Trotz ihrer Häufigkeit und der erheblichen Krankheitslast für Patienten und Gesundheitssystem werden depressive Erkrankungen nach wie vor unzureichend behandelt. Einer amerikanischen Untersuchung zufolge erfährt nur etwa jeder zweite Betroffene „irgendeine“ Behandlung, lediglich $22 \%$ werden adäquat therapiert. „Zum einen“, so Kasper, „ist die Erkrankung weiterhin stigmatisiert, zum anderen werden bei vielen Patienten bestehende körperliche Beschwerden oft nicht mit der Depression in Zusammenhang gebracht." Nach den S3-Leitlinien der Fachgesellschaften gelten Symptomreduktion, Verringerung der Suizidgefahr und vollständige Remission als primäre Behandlungsziele. In der Akuttherapie zieht Kasper eine intensive individualisierte Behandlung mit Antidepressiva dem „Watchful waiting" vor. Studien zufolge verbessern moderne SSN-
RI wie Duloxetin (Cymbalta ${ }^{\circledR}$ ) auch die körperlichen Symptome einer Depression. Entscheidend sei, dass eine begonnene und erfolgreiche Pharmakotherapie ausreichend lange durchgeführt wird: bei Ersterkrankungen über vier bis neun Monate, bei Rezidiven mindestens zwei Jahre, häufig sogar lebensbegleitend. „Die Rezidivprophylaxe“, so Kasper, „ist umso wichtiger, je mehr depressive Episoden in der Vergangenheit aufgetreten sind und je kürzer die symptomfreien Intervalle waren. “ Frühe Response und Remission seien die wichtigsten Prädiktoren für den Therapieerfolg.

Gegenüber dem „Mythos Psychotherapie“ bei Depressionen äußerte sich der Psychiater zurückhaltend. „Die wertvollste Psychotherapie ist, wenn ich mit dem Patienten spreche und ihn ausführlich über seine Erkrankung und die möglichen Behandlungen aufkläre“, ist Kasper überzeugt.

Dr. Martina-Jasmin Utzt

Symposium „Psychische Erkrankungen im Blickpunkt von Forschung, Klinik und Gesundheitsökonomie - Wo steht der Patientennutzen?", Berlin 25.11.2011

Veranstalter: Lilly Deutschland 